\title{
Specific frequency bands of amplitude low-frequency fluctuations in memory-related cognitive impairment: predicting Alzheimer's disease
}

\author{
Yin Tian ${ }^{1 *}$, Zechao Ding ${ }^{1}$, Kin Yip Tam ${ }^{3}$, Zhongyan Wang ${ }^{1}$, Huiling Zhang ${ }^{1}$, Dechun \\ Zhao $^{1}$, Yi Zhao ${ }^{1}$, Wei Xu ${ }^{1}$, and Shuxing Zheng ${ }^{2}$; for the Alzheimer's Disease \\ Neuroimaging Initiative ${ }^{\zeta}$ \\ ${ }^{1}$ Bio-information College, ChongQing University of Posts and Telecommunications, ChongQing 400065, China \\ ${ }^{2}$ Colleg of Computer Science and Technology, ChongQing University of Posts and Telecommunications, ChongQing \\ 400065, China \\ ${ }^{3}$ Faculty of Health Sciences, University of Macau, Macau, China \\ ${ }^{\prime}$ It is noted that the data used in this article were obtained from the Alzheimer's Disease Neuroimaging Initiative \\ (ADNI) database (adni.loni.ucla.edu). As such, the investigators within the ADNI contributed to the design and \\ implementation of ADNI and/or provided data but did not participate in analysis or writing of this report. A complete \\ listing of $A D N I$ investigators can be found at: http://adni.loni.ucla.edu/wp-content/uploads/how to apply/ADNI \\ Acknowledgement List.pdf.
}

*Corresponding Author: E-mail: tiany20032003@163.com; Tel.: +86-23-62460536; Fax: +86-23-62460536

Received: August 09, 2015; Revised: August 12, 2015; Published: September 05, 2015

\begin{abstract}
Resting-state functional magnetic resonance imaging was utilized to measure the amplitude low frequency fluctuations (ALFF) in human subjects with Alzheimer's disease (AD) and normal control (NC). Two specific frequency bands (Slow5: 0.01-0.027 Hz and Slow4: 0.027-0.073 Hz) were analysed in the main cognitive control related four subregions of the right ventral lateral prefrontal cortex (VLPFC), i.e. IFJ, posteriorVLPFC, mid-VLPFC, and anterior-VLPFC. Differences in ALFF values between the AD and the NC group were found throughout the subregions of the right VLPFC. Compared to normal control group, decreased ALFF values were observed in AD patients in the IFJ (in two given frequency bands), and the mid-VLPFC (in Slow5). In contrast, increased ALFF valued were observed in AD patients in the posterior-and anteriorVLPFC (in both Slow5 and Slow4), and also in the mid-VLPFC in Slow4. Moreover, significant ALFF differences between the IFJ and three other subregions of the right VLPFC were found. Furthermore, ALFF values in the right VLPFC showed significant correlations with the time course of disease. Taken together, our findings suggest that $A D$ patients have largely abnormalities in intrinsic neural oscillations which are in line with the $A D$ pathophysiology, and further reveal that the abnormalities are dependent on specific frequency bands. Thus, frequency-domain analyses of the ALFF may provide a useful tool to investigate the AD pathophysiology.
\end{abstract}

\section{Keywords}

AD; ALFF; VLPFC; rs-fMRI; top-down modulation

\section{Introduction}

Alzheimer's disease $(A D)$ is a neurodegenerative disorder leading to cognitive deficits. Though the amyloid- $\beta(A \beta)$ peptides plague aggregation and tau hyperphosphorylation are thought to be two main 
causal roles in the pathogenesis of $A D$ [1-2], the underlying neural mechanisms remain uncovered.

Previous neural imaging studies using positron emission tomography (PET) and single-photon emission (SPE) have found that $A D$ patients show abnormally low resting cerebral blood flow and a decreased cerebral metabolic rate for glucose in the widespread brain regions, including the temporal, parietal, and prefrontal cortex [3-6]. Resting-state functional magnetic resonance imaging (rs-fMRI) has also been considered for development as a biomarker and analytical tool for brain diseases evaluation such as $A D$ and PD. The spontaneous low-frequency fluctuations of the blood oxygen level dependent (BOLD) in rsfMRI were thought to reflect spontaneous neuronal activity [7-9]. The amplitude of low frequency fluctuations (ALFF) of BOLD signals, which were believed to be physiologically meaningful, was proposed to assess the amplitude of resting state spontaneous brain activity [10]. Using resting-state spontaneous brain activity technology, researchers found that AD patients were abnormalities of LFF in many brain regions including the hippocampus, the medial and lateral temporal lobes, the medial parietal lobe, the posterior cingulated cortex (PCC) and the medial and superior prefrontal cortex (MPFC) [11-15].

The function of the right ventral lateral prefrontal cortex (VLPFC) is crucial to the goal-directed cognition (i.e., top-down control), involving attention re/orienting, cognitive control, working memory, motor inhibition, and action updating [16-19]. More research evidence indicated that the right VLPFC could be divided into four subregions according to their cognitive function. However, few researches emphasize the changes of the VLPFC in AD patients though AD related disease could lead to learning and memory impairments, aprosexia, and executive dysfunction. In this study, we proposed that individual differences in AD might be captured based on spontaneous neuronal activity in the functional subregions of the VLPFC related to cognitive impairment identified by the ALFF of BOLD signals. Gray matter (GM) related fluctuation amplitudes mainly occurred in two frequency bands, i.e. 0.01-0.027 Hz (Slow5) and 0.027$0.073 \mathrm{~Hz}$ (Slow4). And these two given frequency bands contribute differently to the ALFF [12,20-21], suggesting that individual frequency bands could link to specific characteristics [22]. To validate the hypothesis underlying AD-related ALFF, the characteristic patterns of the changes of ALFF in AD patients will be compared with normal control subjects, especially Slow5 and Slow4. We aim to evaluate if the ALFF techniques could help us to reveal this intrinsic relationship, which may provide a foundation for further investigations of neural cognitive processes in $A D$.

\section{Experimental Methods}

\section{Subjects}

Twenty two right-handed subjects with $A D$ group ( 8 males and 3 females, mean $\pm S D=69.7 \pm 2.4$ years) and $N C$ group (11 males mean $\pm S D=48.5 \pm 8.4$ years) were selected in the current study. All subject data were obtained from the Alzheimer's disease neuroimaging initiative (ADNI, http:// www.loni.ucla.edu/ADNI)

\section{Data acquisition and analysis}

Three-dimensional (3D)-T1 was acquired using Philips Healthcare 3T scanner. High-resolution volumetric T1-weighted spoiled gradient-recalled (SPGR) images were generated for each subject (TR=3000ms, $\mathrm{TE}=30 \mathrm{~ms}$, flip angle $=80^{\circ}$, Slice thickness $=3.3 \mathrm{~mm}, \mathrm{FOV}=24 \times 24 \mathrm{~cm}^{2}, 64 \times 64$ matrix size, 6720 slices).

Data preprocessing were carried out using SPM8 (http://www.fil.ion.ucl.ac.uk/spm), which included acquisition times correction, motion correction, image segmentation, and spatially smooth. Then DPARSF 
[23] was used to analyses the ALFF. Briefly, we converted data from time-domain to frequency-domain using a Fast Fourier Transform (FFT). The square root of the power spectrum was computed and then averaged frequency ranged from $0.01 \mathrm{~Hz}$ to $0.08 \mathrm{~Hz}$ at each voxel. Slow5 (0.01-0.027 Hz) and Slow4 (0.027$0.073 \mathrm{~Hz}$ ) were selected to analyze. This averaged square root was taken as the ALFF. To reduce the global effects of variability across participants, the ALFF of each voxel was divided by the global mean ALFF value for each subject, resulting in a relative ALFF.

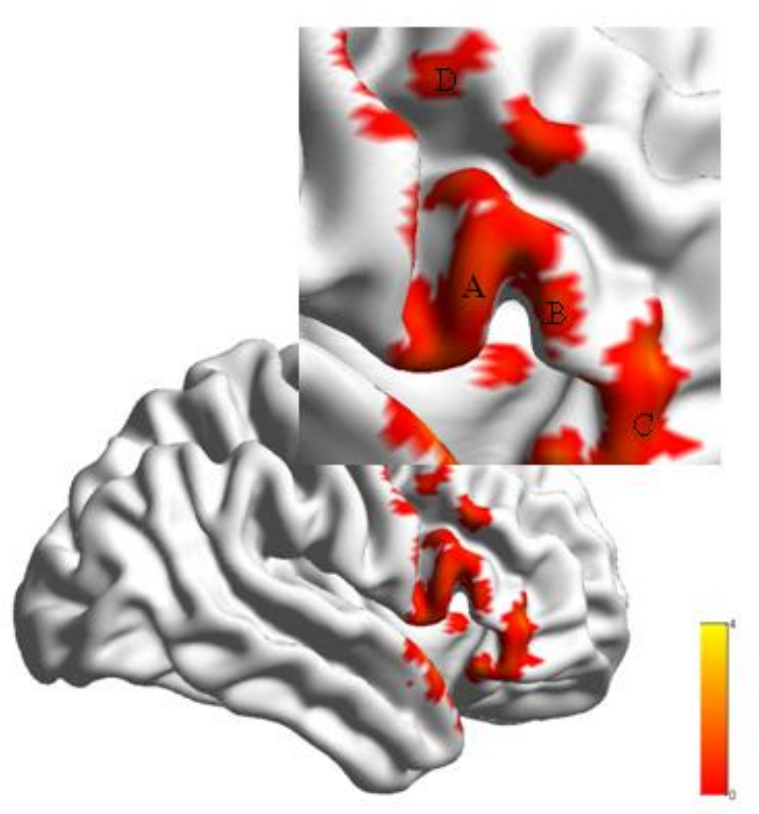

$A D$

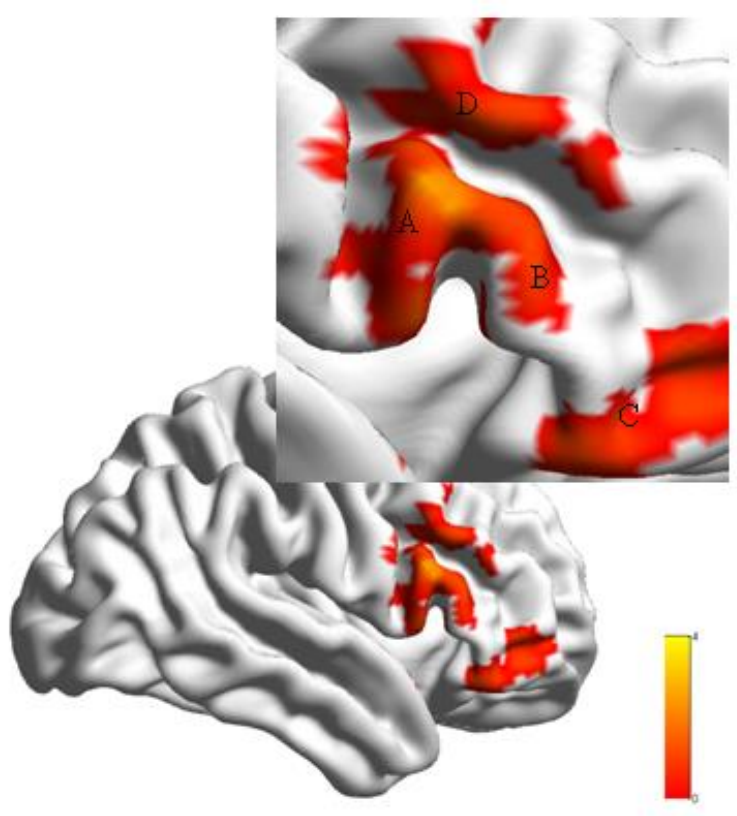

NC

Figure 1. The t-score ALFF maps of $A D$ and NC groups in ROls. ROls: regions of interesting; $A D$ : Alzheimer's disease; NC: normal control; VLPFC: Ventral lateral prefrontal cortex; A: Posterior-VLPFC; B: Middle-VLPFC; C: Anterior-VLPFC; and D: IFJ, inferior frontal junction.

To explore the within-group ALFF patterns, one-sample t-test within the group were performed on the individual ALFF maps in a voxel-wise way for each group. Two-sample t-test among groups, i.e. AD group vs. normal control (NC) group, was conducted. Pearson's correlation coefficient was implemented to analyze the correlations among the mean ALFF and the time course of disease with patients. The statistical threshold was set at $p<0.05$.

For regions of interest (ROIs), i.e. the ventrolateral prefrontal cortex (VLPFC) was divided four functional subregions. Posterior region is the most caudal extent as shown in Fig. 1-region A. Middle region is rostral to the ascending ramus as shown in Fig. 1-region B. Anterior region is the horizontal ramus of the lateral sulcus as shown in Fig. 1-region C. The most posterior and superior region is referred to the inferior frontal junction (IFJ) as shown in Fig. 1-region D.

\section{Results and Discussion}

Figure 1 shows the ALFF maps of AD and NC groups in ROIs, i.e. the four functional subregions in the right ventral lateral prefrontal cortex. The detail information of regions-related $\mathrm{MNI}$ coordinates is shown in Table 1. The ALFF values of each subject including both AD (Red circles) and NC (green circles) were shown in Figure 2. A Multi-ANOVA statistical analysis within factors (regions: posterior-VLPFC vs. midVLPFC vs. anterior-VLPFC vs. IFJ; frequency band: Slow5 vs. Slow4) and between groups (AD vs. NC) was 
conducted. It was found that only the main effect of regions was marginal significant $(F=2.722, P=0.069$, Greeenhour-Geisser correction). Further t-test measurement showed that the ALFF in the IFJ was significantly different in the other regions (posterior-VLPFC: $\mathrm{P}=0.025$; mid-VLPFC: $\mathrm{P}=0.007$; anterior-VLPFC: $P=0.052$, marginal). No any conditions achieve significant differences (all ps $>0.05$ ) between $A D$ group and NC group. Pearson correlation was adopted to measure the relation between time course of disease and ALFF for each of the ROls within the right VLPFC (Fig. 1). As shown in Figure 3, a significant correlation between course of disease and the ALFF of the subregions in the right VLPFC were identified (Negative correlation: regions including A, B, and D in Slow5, and A in Slow4; Positive correlation: D region in Slow4). Apart from that, no other significant relationship was found.

Table 1. Regions showing ALFF differences among the AD and the NC with MNI coordinates.

\begin{tabular}{lccccc} 
& & \multicolumn{3}{c}{ MNI coordinates } & \\
\cline { 3 - 5 } Regions & Group & $\mathrm{x}$ & $\mathrm{y}$ & $\mathrm{z}$ & Brodmann area \\
\hline Frontal_Inf_Oper_R (A) & $\mathrm{AD}$ & 57.7 & 16.1 & 12.2 & 44 \\
Frontal_Inf_Tri_R (B) & $\mathrm{AD}$ & 56.41 & 27.27 & 8.891 & 45 \\
Frontal_Inf_Orb_R (C) & AD & 52.54 & 37.64 & -3.39 & 45 \\
Frontal_Inf_Oper_R (D) & AD & 44.65 & 12.32 & 38.79 & 44 \\
Frontal_Inf_Oper_R (A) & NC & 57.9 & 16.38 & 14.23 & 44 \\
Frontal_Inf_Tri_R (B) & NC & 55.14 & 30.13 & 10.47 & 45 \\
Frontal_Inf_Orb_R (C) & NC & 49.48 & 38.26 & -9.554 & 44 \\
Frontal_Inf_Oper_R (D) & NC & 46.95 & 20.18 & 31.16 & 44 \\
\hline
\end{tabular}

Ps. Frontal_Inf_Oper_R: right inferior frontal operculum; Frontal_Inf_Tri_R: right inferior frontal operculum; Frontal_Inf_Orb_R: right inferior frontal operculum. A: posterior-VLPFC; B: mid-VLPFC; C: anterior-VLPFC; D: IFJ.

Slow5: $0.01-0.027 \mathrm{~Hz}$
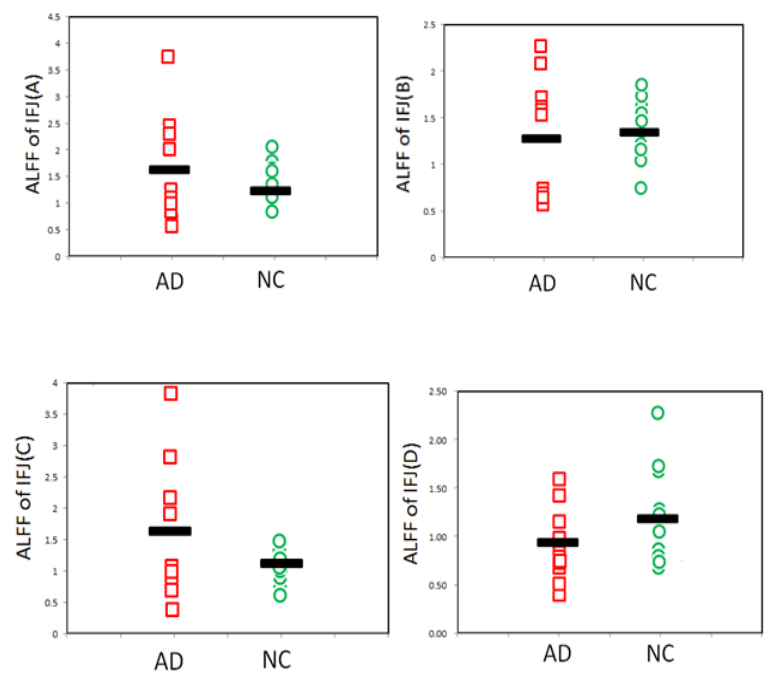

Slow4: 0.027-0.073
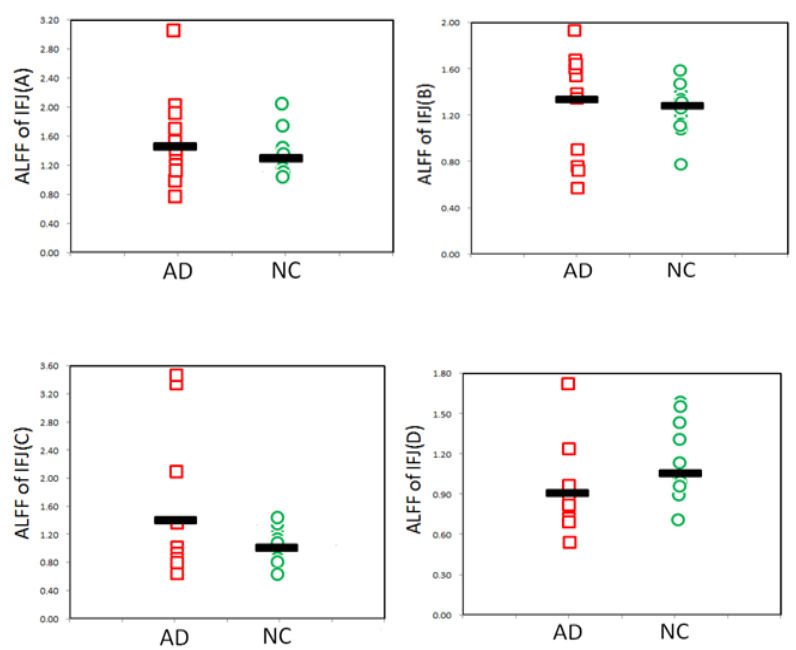

Figure 2. The ALFF values of $A D$ and NC groups in ROIs. ROls: regions of interesting; $A D$ : Alzheimer's disease; NC: normal control; VLPFC: Ventral lateral prefrontal cortex; A: Posterior-VLPFC; B: Middle-VLPFC; C: AnteriorVLPFC; and D: IFJ, inferior frontal junction.

In the present study the $A D$ related changes in the intrinsic brain activity was correlated to the individual ALFF values of rs-fMRI signals in the functional subregions of the right VLPFC (i.e., posterior- 
VLPFC, mid-VLPFC, anterior-VLPFC, and IFJ) and then the associated course of disease. We found that there were differences in ALFF values between the $A D$ and the NC group throughout the subregions of the right VLPFC. Decreased ALFF values were observed in AD patients in the IFJ (in both Slow5 and Slow4), and the mid-VLPFC (in Slow5). In contrast, increased ALFF valued were observed in AD patients in the posteriorand anterior-VLPFC (in both Slow5 and Slow4), and also in the mid-VLPFC in Slow4. Moreover, significant ALFF differences between the IFJ and three other subregions of the right VLPFC were found. Further analysis revealed that the ALFF value for the right VLPFC can be applied to differentiate AD patients from normal controls with a high sensitivity. We also found that ALFF values in the right VLPFC showed significant correlations with the time course of disease.

Slow5: $0.01-0.027 \mathrm{~Hz}$

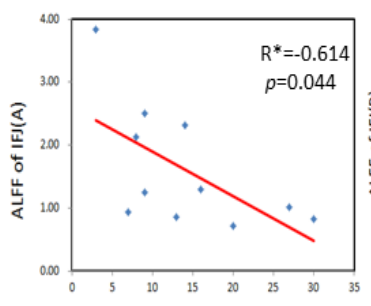

Course of disease

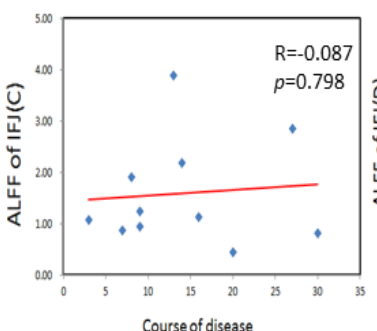

Course of disease

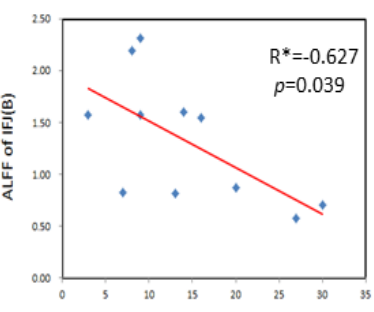

Course of disease

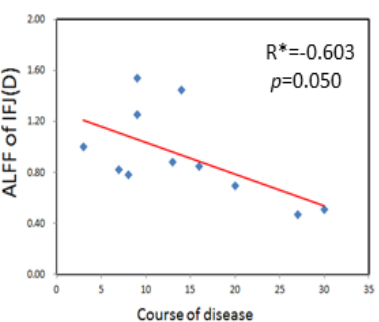

Slow4:0.027-0.073Hz
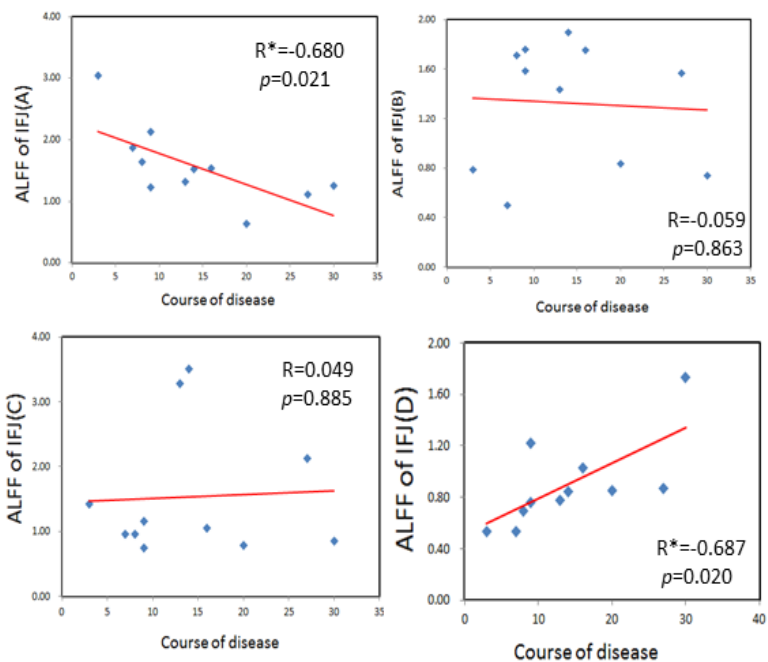

Figure 3. Relationships between the ALFF values and the course of disease (month) in ROls. ROls: regions of interesting; AD: Alzheimer's disease; NC: normal control; VLPFC: Ventral lateral prefrontal cortex; A: PosteriorVLPFC; B: Middle-VLPFC; C: Anterior-VLPFC; and D: IFJ, inferior frontal junction. * :significant level.

The broader evidence indicated that specific forms of cognitive control are linked with distinct functional subregions of the right VLPFC [16-17]. The IFJ supports the detection of behavioral relevant stimuli. The top-down modulation mediated by the IFJ plays a causal role between early attentional processes and subsequent memory performance [16-17, 24-25]. In our current study, decreased ALFF in several subregions in the right VLPFC including IFJ (in both Slow5 and Slow4) was in accordance with previous studies that this region had structural and functional abnormalities. Furthermore, we noticed that there were significant negative correlations between the ALFF of the IFJ in Slow5 and the course of disease, indicating that the decline ALFF for this region along with the course of disease were associated with memory-related cognitive impairment in these patients. In contrast, the significant positive correlation between the ALFF of the IFJ in Slow4 and the course of disease, suggesting the existence of compensation process for cognitive resources [12]. Other subregions in right VLPFC are related to motor control, i.e. posterior-VLPFC is activated by the active plan updating and mid-VLPFC is responsible for decision uncertainty. Anterior-VLPFC encoding higher-order goals usually bridge the longer time spans, and the midVLPFC (in Slow5) [16]. In our study, the significant negative correlations between the ALFF of the posteriorVLPFC and the course of disease, suggest that the longer time after the onset of disease accompanied with the weaker motor control. 


\section{Conclusions}

In summary, we found that AD patients had abnormal ALFF in the subregions of right VLPFC, including IFJ, posterior-VLPFC, mid-VLPFC, and anterior-VLPFC. These findings were similar with previous studies in $A D$. Our findings indicated that the abnormal intrinsic neural oscillations in $A D$ present different neural patterns in different frequency bands, predicting the memory related impairment with the course of disease. Further investigations are important to reveal how they relate to the clinical and pathological correlations of ALFF changes in AD.

Acknowledgements: This study is supported by the National Nature Science Foundation of China (\#31100745), the CQCBE Project KJ110502 and the Science and Technology Development Fund, Macao S.A.R (FDCT) (project reference no.: 118/2013/A3).

\section{References}

[1] J. Hardy, Neuron 52 (2006) 3-13.

[2] Z. S. Khachaturian, Arch. Neurol. 42 (1985) 1097-1105.

[3] A.L. Bokde, P. Pietrini, V. Ibáñez, M.L. Furey, G.E. Alexander, N.R. Graff-Radford, S.I. Rapoport, M.B. Schapiro, B. Horwitz, Arch. Neurol. 58 (2001) 480-486.

[4] M.J. de Leon, A. Convit, O.T. Wolf, C.Y. Tarshish, S. DeSanti, H. Rusinek, W. Tsui, E. Kandil, A.J. Scherer, A. Roche, A. Imossi, E. Thorn, M. Bobinski, C. Caraos, P. Lesbre, D. Schlyer, J. Poirier, B. Reisberg, J. Fowler, Proc. Natl. Acad. Sci. USA 98 (2001) 10966-10971.

[5] K. Herholz, E. Salmon, D. Perani, J-C. Baron, V. Holthoff, L. Frölich, P. Schönknecht, K. Ito, R. Mielke, E. Kalbe, G. Zündorf, X. Delbeuck, O. Pelati, D. Anchisi, F. Fazio, N. Kerrouche, B. Desgranges, F. Eustache, B. Beuthien-Baumann, C. Menzel, J. Schröder, et al., Neuroimage 17 (2002) 302-316.

[6] E. Salmon, F. Collette, C. Degueldre, C. Lemaire, G. Franck, Hum. Brain Mapp. 10 (2000) 39-48.

[7] B. Biswal, F.Z. Yetkin, V.M. Haughton, J.S. Hyde, Magn. Reson. Med. 34 (1995) 537-541.

[8] P. Fransson, Hum. Brain Mapp. 26 (2005) 15-29.

[9] V. Kiviniemi, J. Jauhiainen, O. Tervonen, E. Pääkkö, J. Oikarinen, V. Vainionpää, H. Rantala, B. Biswal, Magn. Reson. Med. 44 (2000) 373-378.

[10] Z. Wang, C. Yan, C. Zhao, Z. Qi, W. Zhou, J. Lu, Y. He. K Li, Hum. Brain Mapp. 32 (2011) 1720-1740.

[11] F. Bai, Neurosci. Lett. 438 (2008) 111-115.

[12] Y. He, L. Wang, Y. Zang, L. Tian, X. Zhang, K. Li, T. Jiang, Neuroimage 35 (2007) 488-500.

[13] Y. Han, J. Wang, Z. Zhao, B. Min, J. Lu, K. Li, Y. He, J. Jia, Neurolmage 55 (2011) 287-295.

[14] K. Wang, M. Liang, L. Wang, L. Tian, X. Zhang, K. Li, T. Jiang, Hum. Brain Mapp. 28 (2007) 967-978.

[15] H.Y. Zhang, S.-J. Wang, J. Xing, B. Liu, Z.-L. Ma, M. Yang, Z.-J. Zhang, G.-J. Teng, Behav. Brain Res. 197 (2009) 103-108.

[16] B.J. Levy, A.D. Wagner, Annals of the New York Academy of Sciences 1224 (2011) 40-62.

[17] Y. Tian, S. Liang, D. Yao, Neurosci. Bull. 30 (2014) 141-152.

[18] Y.Tian, D. Yao, Biol. Psychol. 79 (2008) 171-178.

[19] Y. Tian, S. Liang,Z. Yuan, S. Chen, P. Xu, D. Yao, NeuroReport 25 (2014) 843-847.

[20] X.N. Zuo, A. Di Martino, C. Kelly, Z.E. Shehzad, D.G. Gee, D.F. Klein, F.X. Castellanos, B.B. Biswal, M.P. Milham, Neurolmage 49 (2010) 1432-1445.

[21] A.T. Baria, M.N. Baliki, T. Parrish, A.V. Apkarian, J. Neurosci. 31 (2011) 7910-7919.

[22] G .Buzsáki, A. Draguhn, Science 304 (2004) 1926-1929. 
[23] C. Yan, Y. Zang, Front. Syst. Neurosci. 4 (2010) 13.

[24] A. Gazzaley, A.C. Nobre, Trends Cogn. Sci. 16 (2012) 129-135.

[25] M. D’Esposito, B. R. Postle, Annu. Rev. Psychol. 66 (2015) 115.

(C)2015 by the authors; licensee IAPC, Zagreb, Croatia. This article is an open-access article distributed under the terms and conditions of the Creative Commons Attribution license (http://creativecommons.org/licenses/by/3.0/) (cc)) EY 